\title{
IncHI2 Plasmids Are Predominant in Antibiotic-Resistant Salmonella Isolates
}

\author{
Wenyao Chent, Tingzi Fangt, Xiujuan Zhou, Daofeng Zhang, Xianming Shi and \\ Chunlei Shi*
}

Ministry of Science and Technology-United States Department of Agriculture Joint Research Center for Food Safety, School of Agriculture and Biology, Shanghai Jiao Tong University, Shanghai, China

OPEN ACCESS

Edited by:

Dongsheng Zhou,

Beijing Institute of Microbiology and Epidemiology, China

Reviewed by:

Xian-Zhi Li,

Health Canada, Canada

Pina Fratamico,

United States Department

of Agriculture, Agricultural Research

Service, USA

*Correspondence:

Chunlei Shi

clshi@sjtu.edu.cn

tThese authors have contributed equally to this work.

Specialty section:

This article was submitted to

Antimicrobials, Resistance and Chemotherapy,

a section of the journal

Frontiers in Microbiology

Received: 15 July 2016 Accepted: 20 September 2016 Published: 30 September 2016

Citation:

Chen W, Fang $T$, Zhou $X$, Zhang $D$, Shi $X$ and Shi C (2016) IncHI2

Plasmids Are Predominant in Antibiotic-Resistant Salmonella Isolates. Front. Microbiol. 7:1566. doi: 10.3389/fmicb.2016.01566
The wide usage of antibiotics contributes to the increase in the prevalence of antibioticresistant Salmonella. Plasmids play a critical role in horizontal transfer of antibiotic resistance markers in Salmonella. This study aimed to screen and characterize plasmid profiles responsible for antibiotic resistance in Salmonella and ultimately to clarify the molecular mechanism of transferable plasmid-mediated antibiotic resistance. A total of 226 Salmonella isolates were examined for antimicrobial susceptibility by a disk diffusion method. Thirty-two isolates (14.2\%) were resistant to at least one antibiotic. The presence of plasmid-mediated quinolone resistance (PMQR) genes and $\beta$-lactamase genes were established by PCR amplification. PCR-based replicon typing revealed that these 32 isolates represented seven plasmid incompatibility groups (IncP, HI2, A/C, Flls, FIA, FIB, and I1), and the IncHI2 (59.4\%) was predominant. Antibiotic resistance markers located on plasmids were identified through plasmid curing. Fifteen phenotypic variants were obtained with the curing efficiency of $46.9 \%$ (15/32). The cured plasmids mainly belong to the $\mathrm{HI} 2$ incompatibility group. The elimination of IncHI2 plasmids correlated with the loss of $\beta$-lactamase genes (bla OXA-1 and bla TEM-1) and PMQR genes (qnrA and $\left.\mathrm{aac}\left(6^{\prime}\right)-\mathrm{Ib}-\mathrm{Cr}\right)$. Both IncHI2 and Incl1 plasmids in a S. enterica serovar Indiana isolate SJTUF 10584 were lost by curing. The bla $\mathrm{CMY}_{2}$-carrying plasmid pS10584 from SJTUF 10584 was fully sequenced. Sequence analysis revealed that it possessed a plasmid scaffold typical for Incl1 plasmids with the unique genetic arrangement of IS1294$\triangle \mathrm{ISE} c$ 1-bla $\mathrm{CMY}-2$-b/c-sugE- $\triangle \mathrm{ecnR}$ inserted into the colicin gene cia. These data suggested that IncHI2 was the major plasmid lineage contributing to the dissemination of antibiotic resistance in Salmonella and the activity of multiple mobile genetic elements may contribute to antibiotic resistance evolution and dissemination between different plasmid replicons.

Keywords: Salmonella, antibiotic resistance, replicon typing, plasmid curing, IncHI2, plasmid sequencing

\section{INTRODUCTION}

Salmonella is recognized as the predominant pathogen implicated in bacterial foodborne diseases worldwide (Yang et al., 2016). The emergence of multidrug-resistant (MDR) Salmonella strains due to the prolonged and extensive usage of antibiotics has become a public health issue. Such resistance toward medically significant antimicrobial agents including fluoroquinolones and 
extended-spectrum cephalosporins (ESCs) that are regarded as primary treatment options for bacterial infections will make it difficult for antibiotic therapy due to the reduced efficiency of empirical strategies as well as limited choice of treatment (Wong et al., 2014; Folster et al., 2015).

The dissemination of undesirable antibiotic resistance in Gram-negative bacteria has been largely attributed to the acquisition of multiple plasmid-located antibiotic resistance genes by horizontal gene transfer (HGT; Carattoli, 2013; Wang et al., 2015). The emergence of plasmid-mediated quinolone resistance (PMQR) predominantly encoded by $q n r$ variants, $a a c\left(6^{\prime}\right)-I b-c r$, qepA, and oqxAB genes in Salmonella, has further facilitated selection of high-level chromosomal quinolone resistance via mutations in topoisomerase genes, as well as the wide spread of quinolone resistance via HGT (Jiang et al., 2014; Skov et al., 2015). In addition, the apparent correlation between the PMQR genes (such as $q n r$ or $a a c\left(6^{\prime}\right)-I b-c r$ genes) and extended-spectrum $\beta$-lactamase (ESBL) genes or $a m p C$ genes on the same plasmid and their association with other antibiotic resistance genes have raised particular global concern, since their transmission could be driven by other mobile genetic elements located on plasmids among various bacterial species (Wang et al., 2013; Jiang et al., 2014). Particular plasmid families associate with the emergence and dissemination of specific antibiotic resistance traits with differential prevalence and distribution (Johnson et al., 2007; Carattoli, 2013). Thus, characterization of plasmids based on PCR-based replicon typing (PBRT) is an indispensable part of plasmid epidemiological surveillance enhancing discrimination between Salmonella strains as well as tracing the spread and evolution of antibiotic resistance genes (Wang et al., 2013). Certain replicon types were found to be associated with MDR as well as with bacterial disease outbreaks (Huang et al., 2012). It has been reported that plasmids with IncA/C, B/O, HI1, HI2, I1, N, $\mathrm{F}$, and $\mathrm{P}$ replicons are often associated with MDR in Salmonella, while many of them are found to be co-resident in some MDR Salmonella strains (Poole et al., 2009; Glenn et al., 2013).

Transmissible plasmids contain a full set of conjugationencoding genes facilitating their spread over large taxonomic distances, and they harbor drug-resistance determinants, virulence factors and addiction systems promoting their stability and maintenance in bacterial hosts under different environmental conditions. It is essential to clarify the roles of these genetic elements and the corresponding surrounding genetic structures by plasmid sequencing in order to trace the transmission and persistence of antibiotic resistance (Carattoli, 2013; Wang et al., 2015).

The aim of this study was to screen and characterize plasmid replicon profiles containing PMQR and $\beta$-lactamase genes in Salmonella so as to clarify molecular mechanism of transferable plasmid-mediated antibiotic resistance.

\section{MATERIALS AND METHODS}

\section{Salmonella Isolates}

A total of 226 Salmonella isolates were used in this study, and all the non-susceptible strains are listed in Supplementary
Table S1, of which 39 were food isolates and 39 were clinical isolates. Among these Salmonella isolates, clinical isolates were collected by Shanghai Municipal Center for Disease Control and Prevention and Wuhan Municipal Center for Disease Control and Prevention, while food isolates were collected from beef, poultry, pork, shrimp, vegetables, fresh juice, and shellfish. PCR-based serotyping was performed as previously described (Ranieri et al., 2013). Two different primer pairs and an additional primer pair were used in this study (Supplementary Table S2). The detailed information of these isolates is listed in Supplementary Table S1.

\section{Antimicrobial Susceptibility Testing}

All 226 Salmonella isolates underwent antimicrobial susceptibility testing using the disk diffusion method against a panel of 18 antibiotics, according to the standards and guidelines recommended by the Clinical and Laboratory Standards Institute (CLSI; CLSI, 2013). A total of 18 antibiotic disks (Oxoid Ltd., Basingstoke, UK) that included ampicillin (AMP, $10 \mu \mathrm{g}$ ), piperacillin/tazobactam (TZP, 100/10 $\mu \mathrm{g}$ ), ampicillin/sulbactam (SAM, 10/10 $\mu \mathrm{g}$ ), ceftriaxone (CRO, $30 \mu \mathrm{g}$ ), ceftazidime (CAZ, $30 \mu \mathrm{g})$, cefepime (FEP, $30 \mu \mathrm{g}$ ), cefotetan (CTT, $30 \mu \mathrm{g}$ ), aztreonam (ATM, $30 \mu \mathrm{g})$, cephazolin (CZO, $30 \mu \mathrm{g})$, ciprofloxacin (CIP, $5 \mu \mathrm{g}$ ), imipenem (IPM, $10 \mu \mathrm{g}$ ), amikacin (AMK, $30 \mu \mathrm{g}$ ), gentamicin (GEN, $10 \mu \mathrm{g})$, tobramycin (TOB, $10 \mu \mathrm{g}$ ), ertapenem (ETP, $10 \mu \mathrm{g}$ ), levofloxacin (LEV, $5 \mu \mathrm{g}$ ), nitrofurantoin (NIT, $300 \mu \mathrm{g}$ ), and sulfamethoxazole/trimethoprim (SXT, 23.75/1.25 $\mu \mathrm{g}$ ), were assessed. Escherichia coli ATCC 25922 was used as the control strain. Isolates were defined as MDR if they were resistant to at least three different classes of antibiotics.

\section{Detection of PMQR and $\beta$-Lactamase Genes}

Genomic DNA of Salmonella isolates was purified by the cetyltrimethylammonium bromide (CTAB) method as described elsewhere (Wilson, 2001). The antibiotic-resistant isolates identified by antimicrobial susceptibility testing were screened for the presence of PMQR determinants ( $q n r A, q n r B, q n r S$, qnrC, $\left.q n r D, a a c\left(6^{\prime}\right)-I b-c r\right)$ and $\beta$-lactamase genes (bla $a_{\mathrm{TEM}-1}, b l a_{\mathrm{OXA}-1}$, $b l a_{\mathrm{PSE}-1}$, and $\left.b l a_{\mathrm{CMY}-2}\right)$ by simplex PCR amplification. Primers targeting above antibiotic resistance genes were listed in Supplementary Table S3.

\section{Plasmid Replicon Typing}

Plasmid incompatibility (Inc) groups were assigned by PBRT using genomic DNA of Salmonella isolates as template. Amplification by PCR was performed with eighteen specific primer pairs designed for FIA, FIB, FIC, HI1, HI2, I1, L/M, N, $\mathrm{P}, \mathrm{W}, \mathrm{T}, \mathrm{A} / \mathrm{C}, \mathrm{K}, \mathrm{B} / \mathrm{O}, \mathrm{X}, \mathrm{Y}, \mathrm{F}$, and FIIA basic replicons, and the corresponding protocol was previously described (Carattoli et al., 2005). Resulting amplicons were sequenced by Shanghai Majorbio Bio-pharm Technology Co., Ltd. Comparative analysis of nucleotide sequences was performed using the BLAST 
program at the National Center for Biotechnology Information (NCBI) site ${ }^{1}$.

\section{Plasmid Curing}

To determine if the antibiotic resistance observed in this study was plasmid-mediated, the identified antibiotic-resistant Salmonella isolates were subjected to a curing treatment using sodium dodecyl sulphate (SDS), according to Tomoeda et al. (1968) with modifications. Briefly, the overnight culture in Luria-Bertani (LB) broth was inoculated (1:10 ${ }^{4}$ dilution) in LB broth containing 5\% SDS and incubated for $48 \mathrm{~h}$ at $44.5^{\circ} \mathrm{C}$ with constant agitation. Aliquots of the cultures were then appropriately diluted and spread on LB agar plates without antibiotics and incubated at $37^{\circ} \mathrm{C}$ for $16-18 \mathrm{~h}$. The resulting single clones were streaked onto two LB agar plates with or without ampicillin. Because ampicillin was the most prevalent among antibiotic-resistant isolates, for the screening convenience of those plasmid-loss mutants, ampicillin was used as the priority selective marker. The LB agar plates were incubated at $37^{\circ} \mathrm{C}$ overnight, and ampicillin-susceptible clones were considered positive for loss of the incompatible plasmid. After treatment with the curing agent, the putative cured derivatives were examined for the antibiotic susceptibility profile using the same set of antibiotics, and for the presence of related antibiotic resistance genes as well as plasmid replicon type by PCR method as described above.

\section{Plasmid Sequencing and Analysis}

The Salmonella isolate SJTUF 10584 identified in this study was chosen as the plasmid donor for its complete loss of plasmids and MDR after one single trial of plasmid curing. The plasmid was obtained through conjugation experiments by the liquid mating assay using rifampin-resistant E. coli $\mathrm{Nk} 5449$ as the recipient strain as previously described (Zurfluh et al., 2014). Transconjugants were selected on LB agar plates containing ampicillin $(100 \mu \mathrm{g} / \mathrm{ml})$ and rifampin $(200 \mu \mathrm{g} / \mathrm{ml})$. Antimicrobial susceptibility tests and antibiotic resistance gene detection were performed to confirm the plasmid transfer, followed by PBRT to test which plasmid and antibiotic resistance markers were transferred.

Plasmid DNA was purified from the transconjugant by the Qiagen Plasmid Midi Kit (Qiagen, Germany) according to the manufacturer's protocol. Plasmid sequencing was performed on the IIIumina Hiseq sequencing platform at the Shanghai Biotechnology Corporation (Shanghai, China). The complete sequence was annotated using the NCBI Prokaryotic Genome Annotation Pipeline (PGAP), followed by manual inspection. Each predicted protein was further confirmed against the NCBI all-protein database using BLASTP ${ }^{1}$. Insertion sequences and repetitive elements were identified using IS finder ${ }^{2}$. DNA sequence comparisons and alignments were performed using $\mathrm{BLAST}^{1}$ and Geneious software (version 9.1.2; Kearse et al., 2012), and a schematic plasmid map was constructed with WinPlas 2.7 software.

${ }^{1}$ http://blast.ncbi.nlm.nih.gov/Blast.cgi

${ }^{2}$ https://www-is.biotoul.fr//

\section{Nucleotide Sequence Accession Number}

The complete sequence of the plasmid pS10584 has been deposited in Genbank/EMBL/DDBJ with accession number KX058576.

\section{RESULTS AND DISCUSSION}

\section{Antimicrobial Susceptibility}

Among 226 Salmonella isolates, 14.2\% (32/226) demonstrated resistance to at least one antibiotic and $11.1 \%(25 / 226)$ were resistant to $\geq 3$ antibiotics. In addition, $4.9 \%(11 / 226)$ showed MDR phenotypes. It's noteworthy that the strain SJTUF 10702 isolated from chicken exhibited strong resistance to 11 antibiotics. In terms of 32 antibiotic-resistant isolates (Figure 1), $25.0 \%(8 / 32)$ presented the quinolone resistance phenotype (LEV or CIP) while $96.9 \%(31 / 32)$ were resistant to the five tested $\beta$-lactams (AMP, SAM, CZO, CRO, and CAZ) alone or in combination. Moreover, resistance to individual agents was most frequently observed to AMP $(31 / 32,96.9 \%)$ and SAM $(26 / 32$, $81.3 \%)$, followed by TOB $(14 / 32,43.8 \%)$ and SXT $(11 / 32,34.4 \%)$. No resistance was detected to CTT, FEP, IPM, and ETP.

\section{Prevalence of PMQR and $\beta$-Lactamase Genes}

Amongst the 32 antibiotic-resistant Salmonella isolates, the PMQR genes $a a c\left(6^{\prime}\right)-I b-c r$ and $q n r A$ were present alone or in combination in $25(78.1 \%)$ and $5(15.6 \%)$ isolates, respectively; three isolates harbored both two determinants (Figure 1). The $q n r B, q n r S, q n r C$, and $q n r D$ genes were not detected in any of the tested isolates. In terms of $\beta$-lactamase genes detected, $75.0 \%$ $(24 / 32)$ were positive for bla $a_{\mathrm{OXA}-1}, 37.5 \%(12 / 32)$ for $b l a_{\mathrm{TEM}-1}$ and $9.4 \%(3 / 32)$ for bla $a_{\mathrm{CMY}-2}$. In addition, seven isolates simultaneously carried bla $a_{\mathrm{OXA}-1}$ and $b l a_{\mathrm{TEM}-1}$, and two carried $b l a_{\mathrm{OXA}-1}$ and $b l a_{\mathrm{CMY}-2}$, while one isolate harbored all these three determinants. No isolates contained bla $a_{\mathrm{PSE}-1}$. Of these 27 PMQR-bearing isolates, 23 isolates $(85.2 \%)$ also carried at least one $\beta$-lactamase gene with the prevalent pattern of $a a c\left(6^{\prime}\right)-I b-c r$ bla $a_{\mathrm{OXA}-1}(12 / 23,52.2 \%)$ and $\operatorname{aac}\left(6^{\prime}\right)-\mathrm{Ib}-\mathrm{cr}-b l a_{\mathrm{OXA}-1}-b l a_{\mathrm{TEM}-1}$ $(4 / 23,17.4 \%)$.

\section{Plasmid Replicon Typing}

Plasmid replicons detected among 32 antibiotic-resistant Salmonella isolates are listed in Figure 1. Overall, seven different replicons of the 18 classic replicon types associated with Enterobacteriaceae were identified in all the isolates except SJTUF 10023 and SJTUF 10054. The two untypeable isolates may possess divergent or novel replicons (Carattoli, 2009). PBRT demonstrated that IncHI2 (19/32, 59.4\%) was the predominant type while the other Inc groups such as $\mathrm{P}(7 / 32,21.9 \%), \mathrm{A} / \mathrm{C}(4 / 32,12.5 \%), \mathrm{I} 1$ (3/32, 9.4\%), $\mathrm{FII}_{\mathrm{S}}$ $(2 / 32,6.25 \%)$, FIA $(1 / 32,3.1 \%)$, and FIB $(1 / 32,3.1 \%)$ were just present in few isolates. Seven isolates were mixed of two replicon types, including the combinations of $\mathrm{HI} 2$ and I1 $(3 / 7,42.9 \%), \mathrm{HI} 2$ and $\mathrm{P}(2 / 7,28.6 \%), \mathrm{HI} 2$ and $\mathrm{A} / \mathrm{C}(1 / 7$, $14.3 \%)$, and FIA and FIB $(1 / 7,14.3 \%)$. Inc groups HI2, I1, 


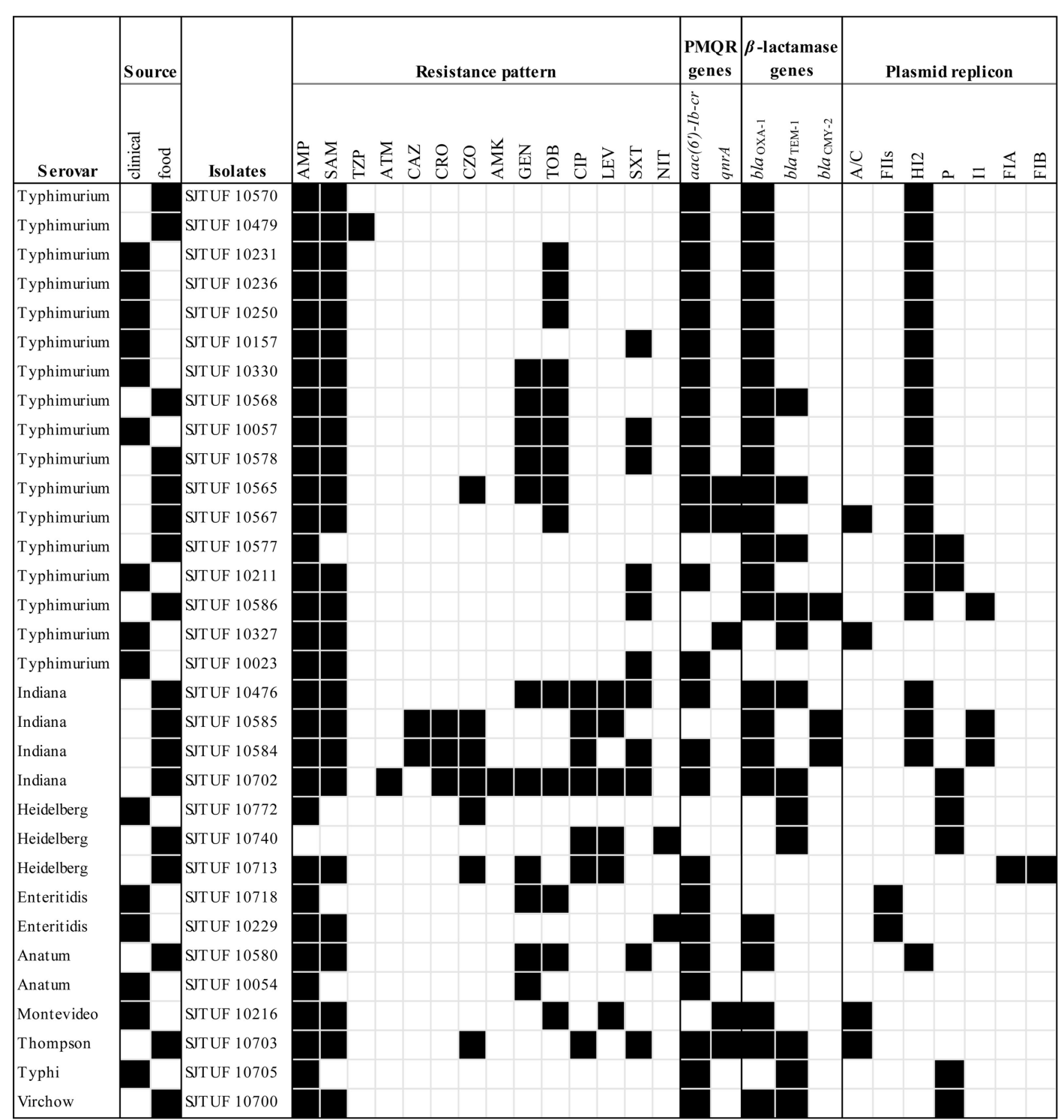

FIGURE 1 | A heat-map summary of the serovars, the sources, the antibiotic resistance phenotypes and genotypes, and the corresponding Inc plasmid type(s) for $\mathbf{3 2}$ drug-resistant Salmonella isolates. Black and white squares denotes the presence and absence of a particular feature, respectively. The antibiotics listed are abbreviated as follows: AMP, ampicillin; TZP, piperacillin/tazobactam; SAM, ampicillin/sulbactam; CRO, ceftriaxone; CAZ, ceftazidime; ATM, aztreonam; CZO, cephazolin; CIP, ciprofloxacin; AMK, amikacin; GEN, gentamicin; TOB, tobramycin; LEV, levofloxacin; NIT, nitrofurantoin; SXT, sulfamethoxazole/trimethoprim.

A/C, P, FIA, and FIB were found to be associated with MDR isolates, in agreement with previous studies (Poole et al., 2009; Glenn et al., 2013). In addition, IncHI2 was the dominant replicon type both among $\mathrm{MDR}$ isolates $(7 / 11,63.6 \%)$ and Salmonella Typhimurium isolates (15/17, 88.24\%). When comparing multiple antibiotic resistance genes with replicon types, $86.7 \%(19 / 22)$ of bla $a_{\mathrm{OXA}-1}$ positive isolates as well as $81.8 \%(9 / 11)$ of bla $a_{\mathrm{OXA}-1}-a a c\left(6^{\prime}\right)-\mathrm{Ib}$-cr positive isolates were carrying IncHI2 plasmids. And all the three bla $a_{\mathrm{CMY}-2}$ positive isolates contained IncHI2 and IncI1 plasmids simultaneously. In addition, $b a_{\mathrm{TEM}-1}$ positive isolates tended to carry the IncHI2 $(5 / 12,41.7 \%)$ and IncP $(6 / 12,50.0 \%)$ plasmids while $q n r A$ positive isolates were likely apt to carry IncA/C plasmids (4/5, $80.0 \%)$.

\section{Plasmid Curing and Antibiotic Resistance Localization}

All the 32 antibiotic-resistant Salmonella isolates underwent plasmid curing using 5\% SDS as well as elevated temperature $\left(44.5^{\circ} \mathrm{C}\right)$ for $48 \mathrm{~h}$ to ensure that the antibiotic resistance profile was related to the plasmid. Fifteen phenotypic variants were obtained after up to 10 trials of curing (Figure 2), and the curing efficiency was $46.9 \%$ (15/32). The cured plasmids mainly belonged to IncHI2 group $(7 / 8,87.5 \%)$, followed by 


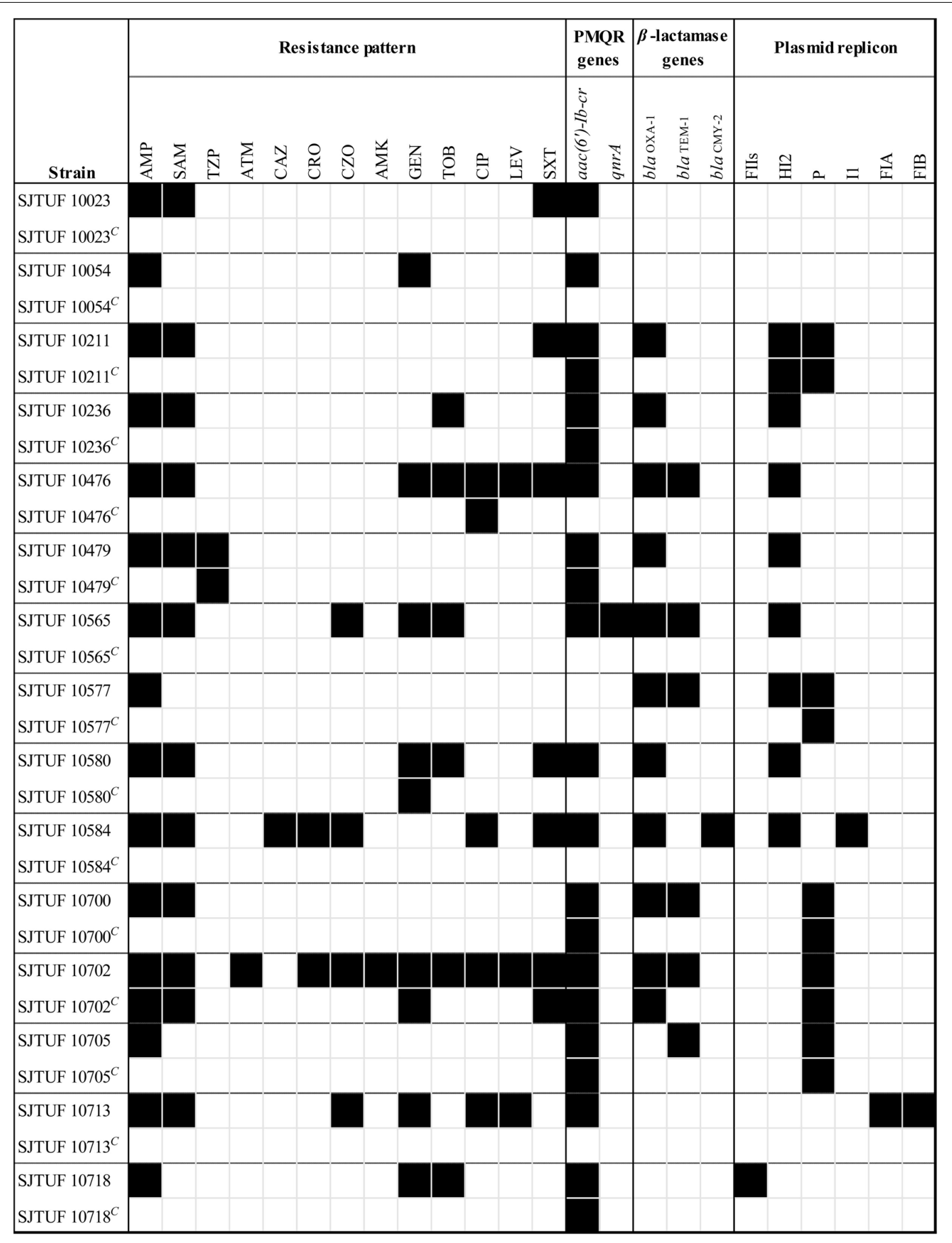

FIGURE 2 | A heat-map showing the changes of Salmonella isolates after plasmid curing based on antibiotic resistance profiles as well as plasmid replicon type(s). "C" denoted the cured variant. Black and white squares denotes the presence and absence of a particular feature, respectively. The antibiotics listed are abbreviated as follows: AMP, ampicillin; TZP, piperacillin/tazobactam; SAM, ampicillin/sulbactam; CRO, ceftriaxone; CAZ, ceftazidime; ATM, aztreonam; CZO, cephazolin; CIP, ciprofloxacin; AMK, amikacin; GEN, gentamicin; TOB, tobramycin; LEV, levofloxacin; SXT, sulfamethoxazole/trimethoprim.

IncF group (3/3,100\%), and IncI1 group (1/1,100\%); however, no strain showed curing of IncP group plasmids $(0 / 5,0 \%)$. When attributing the loss of resistance phenotypes to the loss of plasmids, we could infer that the antibiotic resistant phenotypes AMP-SAM-TOB of SJTUF 10236, AMP-SAM-GENTOB-LEV-SXT of SJTUF 10476, AMP-SAM of SJTUF 10479, AMP-SAM-GEN-TOB-CZO of SJTUF 10565, AMP of SJTUF 10577, and AMP-SAM-TOB-SXT of SJTUF 10580 were all 
related to IncHI2 plasmids while AMP-SAM-CZO-CAZ-CROCIP-SXT of SJTUF 10584 was determined together by IncI1 and IncHI2 plasmids. And the resistant phenotype AMP-SAMCZO-GEN-CIP-LEV of SJTUF 10713 and AMP-GEN-TOB of SJTUF 10718 were both determined by IncF plasmids. In addition, the elimination of IncHI2 plasmids correlated with the loss of $\beta$-lactamase genes (bla $a_{\mathrm{OXA}-1}$ and $b l a_{\mathrm{TEM}-1}$ ) as well as PMQR genes ( $q n r A$ and $\left.a a c\left(6^{\prime}\right)-I b-c r\right)$, and the loss of IncFIA and FIB plasmids resulted in the absence of the $a a c\left(6^{\prime}\right)-I b-c r$ gene. Furthermore, four cured variants (SJTUF $10211^{C}, 10700^{C}, 10702^{C}$ and $10705^{C}$ ) lost antibiotic resistance genes as well as drug resistance phenotypes but still retained the identified plasmids (Figure 2). This may be due to the loss of divergent or novel replicons which were untypeable by the current PBRT scheme. The cured SJTUF $10577^{C}$ lost the AMP resistance phenotype, bla genes, and the IncHI2 plasmid simultaneously, even though it retained the IncP plasmid. The function of IncP plasmids in antibiotic resistance needs further study.

Particular plasmids belonging to the Inc families usually carry multiple physically linked genetic determinants, playing a major role in the diffusion of specific antibiotic resistance genes among different bacteria (Carattoli, 2013; Wang et al., 2013). In this study, we characterized IncHI2 plasmids as the main vehicle in horizontal transfer of $\beta$-lactamase genes (bla ${ }_{\text {OXA }-1}$ and $\left.b l a_{\mathrm{TEM}-1}\right)$ as well as PMQR genes ( $q n r A$ and $\left.a c c\left(6^{\prime}\right)-i b-c r\right)$. The co-existence of $a a c\left(6^{\prime}\right)-I b$-cr with bla $a_{\mathrm{OXA}-1}$, with or without bla $a_{\text {TEM-1 }}$ was also identified. IncHI2 plasmids are known to play a significant role in the acquisition of antibiotic resistance, and they have recently been implicated in the dissemination of genes encoding extended-spectrum $\beta$-lactamase (Cain and Hall, 2012). In contrast, IncA/C plasmids are prevalent in several MDR Salmonella serovars and closely linked to the expansion of MDR in the United States (Folster et al., 2010, 2015). We also discovered the high prevalence of IncHI2 plasmids in $S$. Typhimurium, similar to the report from Li et al. (2013). The identical genetic context of antibiotic resistance genes on IncHI2 plasmids was also detected in S. Indiana in China (Lai et al., 2013) as well as S. Typhimurium in Europe (Campos et al., 2015), suggesting a similar evolutionary origin and highlighting the potentially global spread of IncHI2 plasmids among Salmonella. Furthermore, a similar genetic context of $a a c\left(6^{\prime}\right)-I b-c r$ with bla $a_{\mathrm{OXA}-1}$ was also identified on an IncR plasmid of a K. oxytoca strain in Spain (Ruiz et al., 2011), and an IncN plasmid of an E. coli isolate in Hong Kong (Ho et al., 2013). The similar resistance gene module infers that the transfer of resistanceassociated module was driven by mobile genetic elements like integrons, insertion sequences or transposons between different plasmid replicons, and was probably mediated by IS26 in terms of the above cases (Miriagou et al., 2005; Li et al., 2013; He et al., 2015). Thus, further active surveillance is needed to minimize the spread of particular plasmids such as IncHI2 group to control the dissemination of antibiotic resistance. As mobile genetic elements play a major role in the acquisition and dissemination of antibiotic resistance genes, further study may be required to determine the distinct genetic environment of antibiotic resistance genes on plasmids.

\section{Analysis of pS10584}

The MDR Salmonella enterica serovar Indiana isolate SJTUF 10584 identified in this study was chosen as the plasmid donor. This isolate contains IncHI2 and IncI1 plasmids simultaneously, and these two types of plasmids were easily eliminated after one trial of curing. The plasmid designated as pS10584 was acquired through conjugation experiments recovered from ampicillin and rifampin resistance selection. Replicon typing showed that the only transferred plasmid pS10584 belonged to the IncI1 group and PCR analysis confirmed that it was positive for bla $a_{\mathrm{CMY}-2}$ gene but not for $b l a_{\text {OXA-1 }}$ or $a a c\left(6^{\prime}\right)-I b-c r$ genes. All of the transconjugants shared the same antibiotic resistance pattern of AMP-SAM-CZO-CAZ-CRO.

Complete DNA sequence of pS10584 revealed that it was a circular molecule of 94,697-bp with an average G+C content of $50.0 \%$ and 110 putative CDSs. In general, pS10584 possessed a plasmid scaffold typical for IncI1 plasmids, with important IncI1-associated genetic modules including traABCD regulatory cluster, traLMNOPQRSTUVWXY transfer region, $n i k B$-trbABC region, ssb-psiAB region, and type IV thin pilus formation region pilIJKLMNOPQRSTUV (Figure 3). Besides the approximate 89,866-bp "core genome" region encoding characteristic IncI1 plasmid replication, transfer, maintenance and stability functions, a 4,831-bp accessory module encoding the antibiotic resistance gene as well as flanking insertion sequences was also discovered. BLASTN comparison revealed that the overall genetic organization of pS10584 was very similar to that of another fully sequenced IncI1 plasmid pJIE512b (GenBank accession no. HG970648; Tagg et al., 2014) with high sequence identity (>99\% at nucleotide level) except an inversion within the pilV shufflon and a 2,307-bp deletion (nucleotide $6,685^{\text {th }}$ to $8,991^{\text {nd }}$ of pS10584, containing a truncated colicin gene cia and an intact putative DNA-binding transcriptional regulator gene yagA) in pJIE5112b.

In pS10584, the 4,831-bp antibiotic resistance-associated module comprised 161-bp of the right end of ISEcp1 truncated by IS1294 in the reverse orientation and a 2,823-bp region originating from the Citrobacter freundii chromosome including a bla $a_{\mathrm{CMY}-2}$ gene, with blc (an outer membrane lipoprotein, lipocalin), sugE (a small MDR transporter), and $\triangle e c n R$ (LuxR family transcriptional regulator) genes immediately downstream, followed by a 159-bp fragment of IncA/C ending in GTTC which matched the last 4-bp of terIS of IS1294 (Figure 4). In addition, the 4831-bp bla $a_{\mathrm{CMY}-2}$ segment inserted within the cia gene at the 698-bp from the $5^{\prime}$ end without causing adjacent deletion, followed by the remaining 1,184-bp segment of the cia gene.

A similar genetic context surrounding the $b l a_{\mathrm{CMY}-2}$ gene was identified in an IncI1 plasmid p2735 (KP017243; Yassine et al., 2014) isolated from Klebsiella pneumoniae as well as six IncI1 plasmids isolated from E. coli including two completely sequenced plasmids pJIE512b (HG970648; Tagg et al., 2014), and pR7AC (KF434766; Minoia et al., 2012), and four partially sequenced plasmids pTN38148 (FM246883), pTN10, pTN13, and pTN386 (Verdet et al., 2009) (Figure 4). Compared to pS10584, the $b l a_{\mathrm{CMY}-2}$ insertion was within the yagA gene 16-bp from the $5^{\prime}$ end, removing the rest of the yag $A$ gene, as well as a large part 


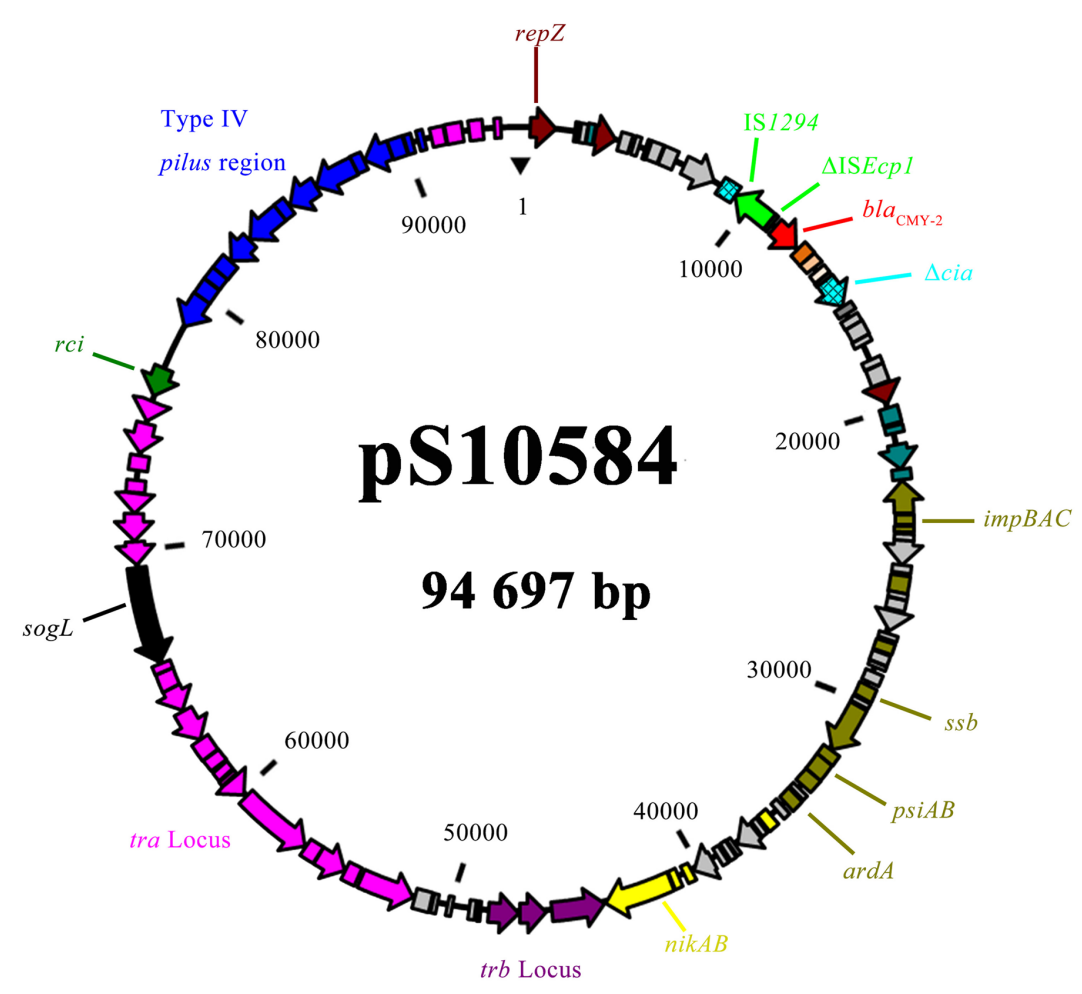

FIGURE 3 | An overview of the bla insertion sequences; red, antibiotic resistance gene; blackish green, partition- or stability-associated genes; army green, transfer-leading region associated genes; pink, tra locus; blue, type IV pilus region; purple, trb locus; and gray, hypothetical protein.

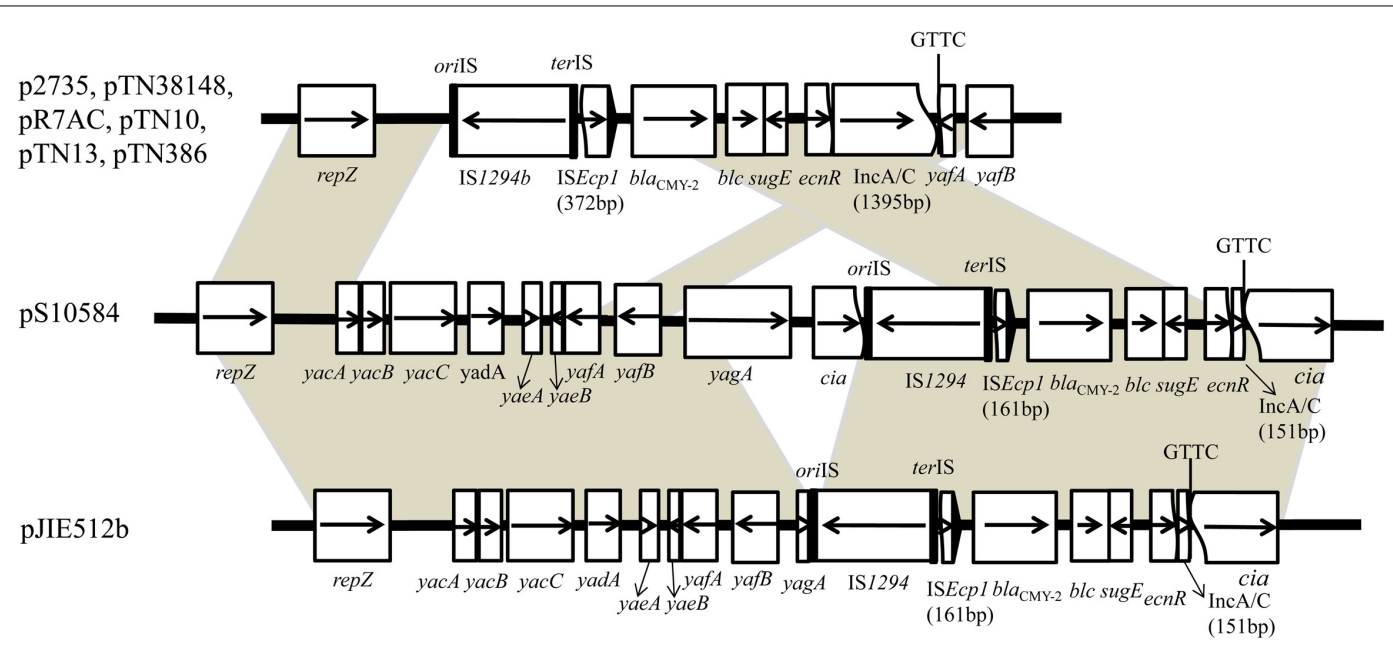

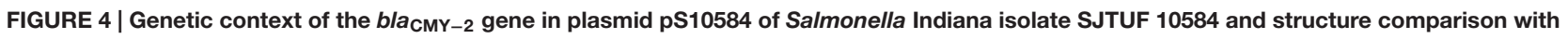
plasmids p2735 from Klebsiella oxytoca and pTN38148, pTN10, pTN13, pTN386, pR7AC, pJIE512b from Escherichia coli. Regions of > 99\% homology are marked by gray shading.

of the cia gene, leaving the same part of the cia gene in the $3^{\prime}$ end followed by the same 159-bp IncA/C backbone ending in GTTC in pJIE512b. Whereas, the flanking fragments of $b l a_{\mathrm{CMY}-2}$ gene in other six IncI1 plasmids (pTN10, pTN13, pTN386, pTN38148, $\mathrm{p} 2735$, and $\mathrm{pR7AC}$ ) were much more different from that in
pS10584, including an IS1294-like element designated as IS1294b (Yassine et al., 2014), followed by a 372-bp truncated ISEcp1, the identical 2,823-bp region of the $C$. freundii chromosome that contained $b l a_{\mathrm{CMY}-2}$ and the additional 1395-bp IncA/C backbone also ending in GTTC (Figure 4). This bla $a_{\mathrm{CMY}-2}$ 
insertion was downstream of the repZ gene, removing a larger adjacent part and truncating the $3^{\prime}$ end of yaf $A$ followed by yaf $B$.

Although $S$. Indiana frequently exhibited MDR phenotypes, very little research has been done to clarify the molecular mechanisms conferring its MDR or the transferability of the resistance properties (Lai et al., 2013). Among ampC-like genes, the $b l a_{\mathrm{CMY}-2}$ gene which probably originated from $C$. freundii, is the most prevalent gene worldwide conferring resistance to clinically important $\beta$-lactam-based compounds particularly the third-generation cephalosporins. (Verdet et al., 2009; Call et al., 2010; Karczmarczyk et al., 2014; Tagg et al., 2014). In this

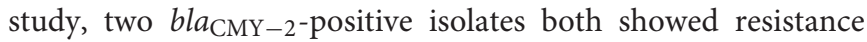
phenotypes against the third-generation cephalosporins (CRO, $\mathrm{CAZ}$, and $\mathrm{CZO}$ ) while the other one was susceptible to them, probably due to the low-level expression or silence of this gene. In previous research, it was observed that resistance to a second generation cephalosporin, cefoxitin in Salmonella isolates harboring bla $a_{\mathrm{CMY}-2}$ (Giles et al., 2004; Martin et al., 2012; Noda et al., 2015). However, none of the three bla $a_{\mathrm{CMY}-2}$-positive isolates was resistant to cefotetan, which is a second-generation cephalosporin like cefoxitin. This was also found in a previous study (Noda et al., 2015). This may also be attributed to the low-level expression or silence of bla $a_{\mathrm{CMY}-2}$ gene.

Among various plasmid types associated with the carriage of the bla $a_{\mathrm{CMY}-2}$ gene, IncIl and IncA/C plasmids are often reported as the predominant carriers (Mata et al., 2012; Bortolaia et al., 2014). Additional research discovered that $b a_{\mathrm{CMY}-2}$ carrying IncA/C plasmids always conferred additional antimicrobial resistance phenotypes such as chloramphenicol and sulfisoxazole, whereas in this study, bla $a_{\mathrm{CMY}-2}$ carrying IncI1 plasmids only conferred a bla $\mathrm{CMY}-2$-associated phenotype, which was also observed in Folster et al. (2011). The bla $a_{\mathrm{CMY}-2}$ genetic context on the IncI1 plasmid identified in this study was IS1294- $\triangle$ ISEcp1bla $a_{\mathrm{CMY}-2}$-blc-sugE- $\triangle e c n R$ followed by 159-bp IncA/C backbone, which was identical to that of pJIE512b from an E. coli isolate (Tagg et al., 2014). The genetic arrangement ISEcp1-bla ${ }_{\mathrm{CMY}-2^{-}}$ blc-sugE-ecnR was conserved on IncA/C plasmids (Verdet et al., 2009; Ye et al., 2016). ISEcp1 was presumed to be responsible for the mobilization of $b l a_{\mathrm{CMY}-2}$ (Verdet et al., 2009) or bla $a_{\mathrm{CTX}-\mathrm{M}-2}$ (Lartigue et al., 2006) as well as its adjacent region from the bacterial chromosome to the IncA/C plasmids by a one-ended mobilization mechanism. On the basis of sequence alignments, it was suggested that mobilization of $b a_{\mathrm{CMY}-2}$ from the IncA/C plasmid to the IncI1 replicon occurred through IS1294-mediated transposition activity (Tagg et al., 2014). The bla $a_{\mathrm{CMY}-2}$ insertion region of pS10584 started in CTTG and ended in GTTC, both of which matched the last 4-bp of oriIS and terIS of IS1294 (Tavakoli et al., 2000; Yassine et al., 2014), implying that IS1294 inserted into ISEcp1 in an IncA/C plasmid and subsequently mobilized the adjacent region, including the $b l a_{\mathrm{CMY}-2}$ segment plus a 159bp IncA/C backbone (Tagg et al., 2014). A similar bla ${ }_{\mathrm{CMY}-2}$ context was also observed in six reported IncI1 plasmids p2735 (Yassine et al., 2014), pR7AC (Minoia et al., 2012), pTN38148, TN10, TN13, and TN386 (Verdet et al., 2009); however, in these plasmids the bla $a_{\mathrm{CMY}-2}$ insertion segment varied in the length of IncA/C backbone and the position that ISEcp1 interrupted by an IS1294-like element designated as IS1294b. And the integration of the $b l a_{\mathrm{CMY}-2}$ gene by IS1294b between IncA/C and IncI1 replicons was experimentally confirmed by Yassine et al. (2014). By comparing the integration sites of bla $a_{\mathrm{CMY}-2}$ insertion segments on these reported IncI1 plasmids, we inferred that the downstream of repZ gene could represent a "hot spot" for the integration of IS1294b element in the IncI1 plasmid scaffold but determination of the "hot spot" for IS1294 may require further study.

Besides the resistance determinants IncI1 plasmids encode, additional factors may be responsible for their prevalent diffusion. The shufflon multiple inversion system on IncI1 plasmids involves four segments separated by seven inverted repeats and a shufflon-specific recombinase (Rci) which acts to catalyze recombination between these repeats (Sampei et al., 2010). This shufflon system is responsible for generating variation in the $\mathrm{C}$ terminus of the pilV tip adhesins of the pili, leading to the specificity of recipient cells in liquid mating (Tagg et al., 2014). The resulting type IV pilus is also a known virulence factor, contributing to adhesion and invasion of Shiga toxinproducing E. coli (Kim and Komano, 1997), which accounts for the prevalent existence of IncI1 plasmids in pathogenic E. coli (Johnson et al., 2007). Thus, the association of epidemic ability as well as resistance determinants may favor the wide dissemination of plasmids belonging to the IncIl group (Carattoli, 2009; Folster et al., 2010).

\section{CONCLUSION}

We screened and characterized antibiotic resistance plasmids from clinical and food Salmonella isolates in this study and discovered that IncHI2 was the major plasmid lineage contributing to the dissemination of antibiotic resistance in Salmonella. We also reported the complete sequence of an IncI1 plasmid harboring an antibiotic resistance-encoding region with the genetic arrangement of IS1294- $\triangle \mathrm{ISEcp} 1-b l a_{\mathrm{CMY}-2}-b l c-$ $\operatorname{sugE-} \triangle e c n R$, interrupting the cia gene from a MDR $S$. Indiana isolate. To our knowledge, this is the first report of this bla ${ }_{\mathrm{CMY}-2}$ context on the IncI1 plasmid in Salmonella. These results revealed that plasmids represent a potential threat for the dissemination of antibiotic resistance since they carry relevant resistance determinants and complete transfer-associated genes. Thus, in addition to phenotypic monitoring of antimicrobial resistance in Salmonella, further active surveillance is needed to minimize the spread of particular plasmids such as those belonging to the IncHI2 group. In addition, investigations of mobile genetic elements that are located on plasmids, such as insertion sequences, integrons, and transposons, may aid in understanding the source and spread of antibiotic resistance.

\section{AUTHOR CONTRIBUTIONS}

WC completed the antimicrobial susceptibility test, PMQR and $\beta$-lactamase gene detection, plasmid replicon typing, plasmid curing, plasmid sequencing, and prepare the manuscript; TF completed the isolate collection, antimicrobial susceptibility test, 
PMQR and $\beta$-lactamase gene detection, plasmid replicon typing, and plasmid curing; XZ helped to finish the isolate collection and antimicrobial susceptibility test; DZ helped to finish the plasmid sequencing and analysis; XS helped to design the project and give some valuable suggestions; and CS designed the project, completed the data analysis, and prepared the manuscript.

\section{ACKNOWLEDGMENTS}

This work was supported by the National Natural Science Foundation of China (grant number 31230058), the National Key

\section{REFERENCES}

Bortolaia, V., Hansen, K. H., Nielsen, C. A., Fritsche, T. R., and Guardabassi, L. (2014). High diversity of plasmids harbouring $b a_{\mathrm{CMY}-2}$ among clinical Escherichia coli isolates from humans and companion animals in the upper Midwestern USA. J. Antimicrob. Chemother. 69, 1492-1496. doi: 10.1093/jac/dku011

Cain, A. K., and Hall, R. M. (2012). Evolution of IncHI2 plasmids via acquisition of transposons carrying antibiotic resistance determinants. J. Antimicrob. Chemother. 67, 1121-1127. doi: 10.1093/jac/dks004

Call, D. R., Singer, R. S., Meng, D., Broschat, S. L., Orfe, L. H., Anderson, J. M.,

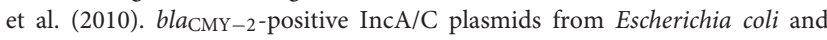
Salmonella enterica are a distinct component of a larger lineage of plasmids. Antimicrob. Agents Chemother. 54, 590-596. doi: 10.1128/AAC.00055-09

Campos, J., Mourão, J., Marçal, S., Machado, J., Novais, C., Peixe, L., et al. (2015). Clinical Salmonella Typhimurium ST34 with metal tolerance genes and an IncHI2 plasmid carrying oqxAB-aac(6')-Ib-cr from Europe. J. Antimicrob. Chemother. 71, 843-845. doi: 10.1093/jac/dkv409

Carattoli, A. (2009). Resistance plasmid families in Enterobacteriaceae. Antimicrob. Agents Chemother. 53, 2227-2238. doi: 10.1128/AAC.01707-08

Carattoli, A. (2013). Plasmids and the spread of resistance. Int. J. Med. Microbiol. 303, 298-304. doi: 10.1016/j.ijmm.2013.02.001

Carattoli, A., Bertini, A., Villa, L., Falbo, V., Hopkins, K. L., and Threlfall, E. J. (2005). Identification of plasmids by PCR-based replicon typing. J. Microbiol. Methods 63, 219-228. doi: 10.1016/j.mimet.2005.03.018

CLSI (2013). Performance Standards for Antimicrobial Susceptibility Testing: Tweenty-Third Informational Supplement M100-S23. Wayne, PA: Clinical and Laboratory Standards Institute.

Folster, J. P., Campbell, D., Grass, J., Brown, A. C., Bicknese, A., Tolar, B., et al. (2015). Identification and characterization of multidrug-resistant Salmonella enterica serotype Albert isolates in the United States. Antimicrob. Agents Chemother. 59, 2774-2779. doi: 10.1128/AAC.05183-14

Folster, J. P., Pecic, G., Bolcen, S., Theobald, L., Hise, K., Carattoli, A., et al. (2010). Characterization of extended-spectrum cephalosporin-resistant Salmonella enterica serovar heidelberg isolated from humans in the United States. Foodborne Pathog. Dis. 7, 181-187. doi: 10.1089/fpd.2009.0376

Folster, J. P., Pecic, G., McCullough, A., Rickert, R., and Whichard, J. M. (2011). Characterization of bla $a_{\mathrm{CMY}}$-encoding plasmids among Salmonella isolated in the United States in 2007. Foodborne Pathog. Dis. 8, 1289-1294. doi: 10.1089/fpd.2011.0944

Giles, W. P., Benson, A. K., Olson, M. E., Hutkins, R. W., Whichard, J. M., Winokur, P. L., et al. (2004). DNA sequence analysis of regions surrounding bla $a_{\mathrm{CMY}-2}$ from multiple Salmonella plasmid backbones. Antimicrob. Agents Chemother. 48, 2845-2852. doi: 10.1128/AAC.48.8.2845-2852.2004

Glenn, L. M., Lindsey, R. L., Folster, J. P., Pecic, G., Boerlin, P., Gilmour, M. W., et al. (2013). Antimicrobial resistance genes in multidrug-resistant Salmonella enterica isolated from animals, retail meats, and humans in the United States and Canada. Microb. Drug Resist. 19, 175-184. doi: 10.1089/mdr.2012.0177

He, S., Hickman, A. B., Varani, A. M., Siguier, P., Chandler, M., Dekker, J. P., et al. (2015). Insertion sequence IS26 reorganizes plasmids in clinically isolated multidrug-resistant bacteria by replicative transposition. mBio 6:e0762-15. doi: $10.1128 / \mathrm{mBio} .00762-15$
Research and Development Program of China (grant number 2016YFD0401102), the Science and Technology Commission of Shanghai Municipality (grant number 14390711900), and the Shanghai Jiao Tong University Agri-X Fund (grant number AgriX2015005).

\section{SUPPLEMENTARY MATERIAL}

The Supplementary Material for this article can be found online at: http://journal.frontiersin.org/article/10.3389/fmicb. 2016.01566

Ho, P.-L., Chan, J., Lo, W.-U., Lai, E. L., Cheung, Y.-Y., Lau, T. C., et al. (2013). Prevalence and molecular epidemiology of plasmid-mediated fosfomycin resistance genes among blood and urinary Escherichia coli isolates. J. Med. Microb. 62, 1707-1713. doi: 10.1099/jmm.0.062653-0

Huang, X.-Z., Frye, J. G., Chahine, M. A., Glenn, L. M., Ake, J. A., Su, W., et al. (2012). Characteristics of plasmids in multi-drug-resistant Enterobacteriaceae isolated during prospective surveillance of a newly opened hospital in Iraq. PLoS ONE 7:e40360. doi: 10.1371/journal.pone.0040360

Jiang, H.-X., Song, L., Liu, J., Zhang, X.-H., Ren, Y.-N., Zhang, W.-H., et al. (2014). Multiple transmissible genes encoding fluoroquinolone and third-generation cephalosporin resistance co-located in non-typhoidal Salmonella isolated from food-producing animals in China. Int. J. Antimicrob. Agents 43, 242-247. doi: 10.1016/j.ijantimicag.2013.12.005

Johnson, T. J., Wannemuehler, Y. M., Johnson, S. J., Logue, C. M., White, D. G., Doetkott, C., et al. (2007). Plasmid replicon typing of commensal and pathogenic Escherichia coli isolates. Appl. Environ. Microbiol. 73, 1976-1983. doi: 10.1128/AEM.00760-07

Karczmarczyk, M., Wang, J., Leonard, N., and Fanning, S. (2014). Complete nucleotide sequence of a conjugative IncF plasmid from an Escherichia coli isolate of equine origin containing $b l a_{\mathrm{CMY}-2}$ within a novel genetic context. FEMS Microbiol. Lett. 352, 123-127. doi: 10.1111/1574-6968. 12364

Kearse, M., Moir, R., Wilson, A., Stones-Havas, S., Cheung, M., Sturrock, S., et al. (2012). Geneious Basic: an integrated and extendable desktop software platform for the organization and analysis of sequence data. Bioinformatics 28, 1647-1649. doi: 10.1093/bioinformatics/bts199

Kim, S.-R., and Komano, T. (1997). The plasmid R64 thin pilus identified as a type IV pilus. J. Bacteriol. 179, 3594-3603.

Lai, J., Wang, Y., Shen, J., Li, R., Han, J., Foley, S. L., et al. (2013). Unique class 1 integron and multiple resistance genes co-located on IncHI2 plasmid is associated with the emerging multidrug resistance of Salmonella Indiana isolated from chicken in China. Foodborne Pathog. Dis. 10, 581-588. doi: 10.1089/fpd.2012.1455

Lartigue, M.-F., Poirel, L., Aubert, D., and Nordmann, P. (2006). In vitro analysis of ISEcp $1 B$-mediated mobilization of naturally occurring $\beta$-lactamase gene bla $a_{\mathrm{CTX}-\mathrm{M}}$ of Kluyvera ascorbata. Antimicrob. Agents Chemother. 50, 1282-1286. doi: 10.1128/AAC.50.4.1282-1286.2006

Li, L., Liao, X., Yang, Y., Sun, J., Li, L., Liu, B., et al. (2013). Spread of $o q x A B$ in Salmonella enterica serotype Typhimurium predominantly by IncHI2 plasmids. J. Antimicrob. Chemother. 68, 2263-2268. doi: 10.1093/jac/dkt209

Martin, L. C., Weir, E. K., Poppe, C., Reid-Smith, R. J., and Boerlin, P. (2012). Characterization of bla $a_{\mathrm{CMY}-2}$ plasmids in Salmonella and Escherichia coli isolates from food animals in Canada. Appl. Environ. Microbiol. 78, 1285-1287. doi: 10.1128/AEM.06498-11

Mata, C., Miró, E., Alvarado, A., Garcillán-Barcia, M. P., Toleman, M., Walsh, T. R., et al. (2012). Plasmid typing and genetic context of ampC $\beta$-lactamases in Enterobacteriaceae lacking inducible chromosomal $a m p C$ genes: findings from a Spanish hospital 1999-2007. J. Antimicrob. Chemother. 67, 115-122. doi: $10.1093 / \mathrm{jac} / \mathrm{dkr} 412$

Minoia, M., Bortolaia, V., Villa, L., Carattoli, A., and Guardabassi, L. (2012). "Complete nucleotide sequences of plasmids p51IT and pR7AC from cefotaxime-resistant Escherichia coli isolated from animal reservoirs in Italy 
and Denmark," in Proceedings of the 4th Central European Symposium on Antimicrobials and Antimicrobial Resistance, Cesar 2012, Primošten.

Miriagou, V., Carattoli, A., Tzelepi, E., Villa, L., and Tzouvelekis, L. S. (2005). IS26-Associated In4-Type integrons forming multiresistance loci in enterobacterial plasmids. Antimicrob. Agents Chemother. 49, 3541-3543. doi: 10.1128/AAC.49.8.3541-3543.2005

Noda, T., Murakami, K., Etoh, Y., Okamoto, F., Yatsuyanagi, J., Sera, N., et al. (2015). Increase in resistance to extended-spectrum cephalosporins in Salmonella isolated from retail chicken products in Japan. PLoS ONE 10:e0116927. doi: 10.1371/journal.pone.0116927

Poole, T. L., Edrington, T. S., Brichta-Harhay, D. M., Carattoli, A., Anderson, R. C., and Nisbet, D. J. (2009). Conjugative transferability of the A/C plasmids from Salmonella enterica isolates that possess or lack bla $a_{\mathrm{CMY}}$ in the $\mathrm{A} / \mathrm{C}$ plasmid backbone. Foodborne Pathog. Dis. 6, 1185-1194. doi: 10.1089/fpd.2009.0316

Ranieri, M. L., Shi, C., Switt, A. I. M., den Bakker, H. C., and Wiedmann, M. (2013). Comparison of typing methods with a new procedure based on sequence characterization for Salmonella serovar prediction. J. Clin. Microb. 51, 17861797. doi: 10.1128/JCM.03201-12

Ruiz, E., Rezusta, A., Sáenz, Y., Rocha-Gracia, R., Vinué, L., Vindel, A., et al. (2011). New genetic environments of $a a c\left(6^{\prime}\right)-I b-c r$ gene in a multiresistant Klebsiella oxytoca strain causing an outbreak in a pediatric intensive care unit. Diagn. Microbiol. Infect. Dis. 69, 236-238. doi: 10.1016/j.diagmicrobio.2010.09.004

Sampei, G.-I., Furuya, N., Tachibana, K., Saitou, Y., Suzuki, T., Mizobuchi, K., et al. (2010). Complete genome sequence of the incompatibility group I1 plasmid R64. Plasmid 64, 92-103. doi: 10.1016/j.plasmid.2010.05.005

Skov, R., Matuschek, E., Sjölund-Karlsson, M., Åhman, J., Petersen, A., Stegger, M., et al. (2015). Development of a pefloxacin disk diffusion method for detection of fluoroquinolone-resistant Salmonella enterica. J. Clin. Microbiol. 53, 3411-3417. doi: 10.1128/JCM.01287-15

Tagg, K. A., Iredell, J. R., and Partridge, S. R. (2014). Complete sequencing of IncI1 sequence type 2 plasmid pJIE512b indicates mobilization of bla $a_{\mathrm{CMY}-2}$ from an IncA/C plasmid. Antimicrob. Agents Chemother. 58, 4949-4952. doi: 10.1128/AAC.02773-14

Tavakoli, N., Comanducci, A., Dodd, H. M., Lett, M.-C., Albiger, B., and Bennett, P. (2000). IS1294, a DNA element that transposes by RC transposition. Plasmid 44, 66-84. doi: 10.1006/plas.1999.1460

Tomoeda, M., Inuzuka, M., Kubo, N., and Nakamura, S. (1968). Effective elimination of drug resistance and sex factors in Escherichia coli by sodium dodecyl sulfate. J. Bacteriol. 95, 1078-1089.

Verdet, C., Gautier, V., Chachaty, E., Ronco, E., Hidri, N., Decré, D., et al. (2009). Genetic context of plasmid-carried bla $a_{\mathrm{CMY}-2}$-like genes in Enterobacteriaceae. Antimicrob. Agents Chemother. 53, 4002-4006. doi: 10.1128/AAC.00753-08
Wang, J., Stephan, R., Karczmarczyk, M., Yan, Q., Hachler, H., and Fanning, S. (2013). Molecular characterization of bla $a_{\mathrm{ESBL}}$-harboring conjugative plasmids identified in multi-drug resistant Escherichia coli isolated from foodproducing animals and healthy humans. Front. Microbiol. 4:188. doi: $10.3389 /$ fmicb.2013.00188

Wang, J., Stephan, R., Zurfluh, K., Hächler, H., and Fanning, S. (2015). Characterization of the genetic environment of bla $a_{\mathrm{ESBL}}$ genes, integrons and toxin-antitoxin systems identified on large transferrable plasmids in multidrug resistant Escherichia coli. Front. Microbiol. 5:716. doi: 10.3389/fmicb.2014. 00716

Wilson, K. (2001). Preparation of genomic DNA from bacteria. Curr. Protoc. Mol. Biol. 00:I:2.4:2.4.1-2.4.5. doi: 10.1002/0471142727.mb0204s56

Wong, M. H., Chan, E. W., Liu, L. Z., and Chen, S. (2014). PMQR genes $o q \times A B$ and $a a c\left(6^{\prime}\right)-I b-c r$ accelerate the development of fluoroquinolone resistance in Salmonella typhimurium. Front. Microbiol. 5:521. doi: 10.3389/fmicb.2014.00521

Yang, X., Huang, J., Wu, Q., Zhang, J., Liu, S., Guo, W., et al. (2016). Prevalence, antimicrobial resistance and genetic diversity of Salmonella isolated from retail ready-to-eat foods in China. Food Control 60, 50-56. doi: 10.1016/j.foodcont. 2015.07.019

Yassine, H., Bientz, L., Cros, J., Goret, J., Bébéar, C., Quentin, C., et al. (2014). Experimental evidence for IS1294b-mediated transposition of the bla $a_{\mathrm{CMY}-2}$ cephalosporinase gene in Enterobacteriaceae. J. Antimicrob. Chemother. 70, 697-700. doi: 10.1093/jac/dku472

Ye, L., Li, R., Lin, D., Zhou, Y., Fu, A., Ding, Q., et al. (2016). Characterization of an IncA/C multidrug resistance plasmid in Vibrio alginolyticus. Antimicrob. Agents Chemother. 60, 3232-3235. doi: 10.1128/AAC.00300-16

Zurfluh, K., Jakobi, G., Stephan, R., Hächler, H., and Nüesch-Inderbinen, M. (2014). Replicon typing of plasmids carrying bla $_{\mathrm{CTX}-\mathrm{M}-1}$ in Enterobacteriaceae of animal, environmental and human origin. Front. Microbiol. 5:555. doi: $10.3389 /$ fmicb. 2014.00555

Conflict of Interest Statement: The authors declare that the research was conducted in the absence of any commercial or financial relationships that could be construed as a potential conflict of interest.

Copyright (c) 2016 Chen, Fang, Zhou, Zhang, Shi and Shi. This is an open-access article distributed under the terms of the Creative Commons Attribution License (CC BY). The use, distribution or reproduction in other forums is permitted, provided the original author(s) or licensor are credited and that the original publication in this journal is cited, in accordance with accepted academic practice. No use, distribution or reproduction is permitted which does not comply with these terms. 